\title{
CT ANGIOGRAPHY EVALUATION OF PERIPHERAL VASCULAR DISEASE AND COMPARISON WITH COLOR DOPPLER ULTRASOUND
}

Sathyabhuwan Singh Netam¹, Rajesh Singh², Sanjay Kumar, Atees Singhal ${ }^{4}$, Vishal Jain ${ }^{5}$

\section{HOW TO CITE THIS ARTICLE:}

Sathyabhuwan Singh Netam, Rajesh Singh, Sanjay Kumar, Atees Singhal, Vishal Jain. "CT Angiography Evaluation of Peripheral Vascular Disease and Comparison with Color Doppler Ultrasound". Journal of Evolution of Medical and Dental Sciences 2015; Vol. 4, Issue 83, October 15; Page: 14504-14514, DOI: 10.14260/jemds/2015/2064

\begin{abstract}
Peripheral vascular disease is narrowing or occlusion of arteries and comprises of atherosclerosis, burger's disease, Renoud's disease and aneurysm. DSA, CT angiography and color Doppler ultrasound are the radiological modalities used for these diseases. Here we compared role of the CT angiography and Color Doppler ultrasonography in detection of peripheral vascular disease. AIMS AND OBJECTIVE: To assess the role of CT angiography in peripheral vascular disease and comparison with color Doppler ultrasound. MATERIAL AND METHOD: we examined 50 patients by CT angiography and color Doppler ultrasound who were suspected for arterial disease. We divided the disease on the ground of severity as follows: No stenosis- grade-0, 0-25\% stenosis-grade-1, 25$50 \%$ stenosis-grade- $2,50-75 \%$ stenosis- grade- 3 and $75-100 \%$ stenosis- grade- 4 . We compared the two modalities in terms of diagnosis and grading of peripheral vascular disease. RESULT: We found Color Doppler ultrasound detected more number of normal vessel segment, grade- 1 and grade- 2 stenosis. In stenosis grade-3 and grade-4 CT angiography and Color Doppler ultrasound are equally sensitive. CONCLUSION: Doppler sonography is better than CT angiography in the diagnosis of early onset (Grade I \& II) cases, with a better assessment of soft plaques, segmental flow and recanalisation in peripheral arterial disease. CDUS is the initial modality of choice in any case of peripheral arterial disease. CT angiography is more accurate in assessing grade- 3 and grade- 4 stenosis. CT angiography exceeds the color Doppler in detecting vascular lesions in cases of gas gangrene and thick patients.
\end{abstract}

KEYWORDS: Color Doppler ultrasound, CT angiography, Peripheral vascular disease.

INTRODUCTION: Peripheral arterial occlusive disease (PAOD) is the atheromatous narrowing or occlusion of an artery or arteries of the leg. Disorder of the system produces complications ranging from minor disabilities to loss of the limb. Therefore, early diagnosis and effective treatment is usually rewarded with good outcome and averts dreadful complications. Conventional digital subtraction angiography (DSA) is considered the gold-standard technique. There are several alternative imaging modalities to DSA, including Duplex ultrasonography (DUS), computed tomography angiography (CTA) and magnetic resonance angiography (MRA). Duplex ultrasonography (DUS) has been used as the initial imaging modality in mild symptomatic PAOD. Thus, conventional B scan Doppler (Colour Doppler scanning) complemented with colour flow imaging was $100 \%$ sensitive in depicting changes according to different aetiologies. Fortunately, with advancement in CT angiography, especially in the multi-detector row technique, larger body volumes can be scanned within short time period at high enough resolution to provide good delineation of arterial flow. This has enabled multi-detector row CT (MDCT) to become a promising modality in lower extremity arterial imaging. In present study, we have made an evaluation of the colour doppler ultrasonographic and CT angiographic characteristics in the peripheral arterial diseases and comparison in between these two modalities. 
OBJECTIVES: To assess the role of CT angiography in peripheral vascular disease and comparison with color Doppler ultrasound.

MATERIAL AND METHODS: This prospective, hospital based was conducted in the Department of Radio-diagnosis, Dr. B.R.A.M Hospital, Raipur, C.G, India. From Feb. 2012 - Sept. 2013 on 50 patients who underwent Colour Doppler Sonography and CT angiography of the extremity based on the different clinical suspicions including both outdoor and indoor patient of Department of Surgery, Medicine and allied, Dr. B.R.A.M Hospital, Raipur. All the patients presenting with the symptoms suggestive of peripheral arterial diseases such as: Numbness, Severe pain, Discolouration, Altered sensorium, Pallor, Pulselessness has included in study. We have not included the Patients unwilling to take part in the study, Patients who leaves the study in between, Patients with iodinated contrast allergy, Patient who has already been diagnosed for the study. USG was carried out with following systems: Prosound SSD4000 (Aloka) with Curved/Convex array transducer 2.5-6 MHz, linear transducer 5-10mHz. Applio MX SSA-780 A (Toshiba) with Curved/Convex array transducer $2.5-\mathrm{mHz}$, linear transducer $5-12 \mathrm{mHz}$. CT examination was carried out with 128 slice CT scan Definition AS Siemens Germany. The aorta, common iliac artery (CIA), external iliac artery (EIA), common femoral artery (CFA), superficial femoral artery (SFA), deep femoral artery (DFA), popliteal artery (PA), anterior tibial artery (ATA), tibio-peroneal trunk (TPT), peroneal artery (PEA) and posterior tibial artery (PTA) were examined in each leg.

The arteries were scanned with a linear, phased-array $(7.5 \mathrm{mHz})$ transducer except aorta which was scanned with convex probe. The patient was placed in supine position for all the arterial segments, except for the PA, ATA, PEA and PTA, which were examined in prone position. In assessing the vessel of interest, the vessel was initially found in B-mode in the axial plane in order to visualize the lumen and the wall of the vessel. Then, the whole vessel was scanned in axial and longitudinal planes by colour-coded duplex doppler followed by spectral tracing of the flow at any suspicious region. A segment was considered as normal when the normal tri-phasic velocity profile with late diastolic reversal was detected. Suspicious areas with a reduced diameter were detected via higher blood velocities, seen as a shift in the colour representing the blood flow on the computer screen. Stenosis was noted when a pre-stenotic, low velocity, mono-phasic flow pattern was detected in the proximal vessel segment. A segment with no flow signal was noted as occluded. The site of maximum stenosis was noted and classified according to the degree of stenosis. MDCT angiography was performed within 1 or 2 days following DUS examination. The examination was performed using a 128 -slice multidetector row CT scanner (Siemens Defination AS).

\section{Scan Parameter}

Phase Arterial

$\begin{array}{ll}\text { Anatomical region } & \text { Coeliac axis to feet } \\ \text { Patient position } & \text { Supine, suspended respiration } \\ \text { Slice collimation } & 2.5 \mathrm{~mm} \\ \text { Slice width } & 3 \mathrm{~mm} \\ \text { Reconstruction interval } & 1.5 \mathrm{~mm} \\ \text { Table feed/rotation } & 15 \mathrm{~mm} \\ \text { Pitch } & 6 \\ \text { Rotation time } & 0.6 \mathrm{~s}\end{array}$


$\mathrm{kV}$

$\mathrm{mA}$

Contrast medium Bolus tracking:

Reconstruction algorithm
80

140

$110-120 \mathrm{ml}$ at $4 \mathrm{ml} \mathrm{s}$

$20 \mathrm{ml} \mathrm{NS}$ at $4 \mathrm{ml} \mathrm{s}$

Soft

Image reconstruction VR and MIP for overview, MPR/CPR for calcific stenosis.

The patients were scanned in supine position from feet to head, from the level of T2 - at the renal artery bifurcation of the descending aorta - to the heel. A 18-gauge or 20-gauge intravenous catheter was placed in an ante-cubital vein and tested by rapid manual injection of $10 \mathrm{ml}$ saline. First, a pre-contrast image was obtained at the level of the distal infrarenal abdominal aorta. On the basis of this transverse image, a region of interest (ROI) was set with an area of $10-15 \mathrm{~mm}^{2}$ in the lumen of the distal infra-renal abdominal aorta. This ROI was used as a reference for the following dynamic measurements of contrast enhancement. A total of $120 \mathrm{ml}$ non-ionic contrast agent and $20 \mathrm{cc} 0.9 \%$ $\mathrm{NaCl}$ was infused with a flow rate of $4 \mathrm{ml} / \mathrm{s}$ using a dual-head pressure injector (Medrad, USA) using a bolus tracking technique and axial sections were obtained.

Using a commercially available workstation (Siemens, Definition AS, Germany), 900-2500 thinsection axial images was obtained. Three-dimensional reconstructions, including maximum-intensity projection (MIP), multiplanar reformatted images (MPR), and volume-rendering (VR) images, were created by two technicians. These additional data were used in the evaluation of lower extremity arterial anatomy.

The axial and reconstructed CT angiographic images were analysed. The vessels of the lower extremities were divided into 11 segments for affected side: Infra renal Aorta, CIA, EIA, CFA, SFA, DFA, TPT, PA, ATA, PTA and PTA. Each segment was evaluated for diagnostic sufficiency such that good differentiation of arteries from background tissue could provide an image quality adequate for confident data collection.

The steno-occlusive lesions were examined primarily by visual inspection as diameter stenoses. The degree of stenosis was detected using electronic callipers. The data for each arterial segment were evaluated and classified as Grade 0/Normal 0\%, Grade I -0-25\%, Grade II - 25-50\%, Grade III - 50-75\% and Grade IV ->75\% (Including occlusion). The dorsalis pedis artery was not included in the evaluation as it was not usually visualized on CTA.

RESULT: The arterial tree was divided into three groups: Aortoiliac, Femoro-popliteal, Tibioperoneal. Total of 150 arterial segments were examined in the aorto-iliac region. The following were the observations (table no-1): On Colour Doppler Ultrasonography we were able to label 46(92\%), $36(72 \%)$ and $39(78 \%)$ cases in the infrarenal aorta, common iliac and external iliac arterial segments respectively as normal(Grade 0 stenosis),while on the CT angiography we were able to quantify $49(98 \%), 39(78 \%)$ and $41(82 \%)$ cases in the infrarenal aorta, common iliac and external iliac arterial segments respectively as normal(Grade 0 stenosis).

Detection rate for grade- 1 and grade- 2 stenosis collectively by CDUS and CTA is $14(9.33 \%)$ and $6(4 \%)$ respectively. The detection rate for the Grade -3 and Grade 4 stenosis was similar for both CDUS and CTA in the mentioned arterial segments of the aorto-iliac group. A total of 200 arterial segments were examined in the femoro-popliteal region. 
The following were the observations (table no-2): On Colour Doppler Ultrasonography no abnormality (Grade 0 stenosis) detected in $42(84 \%), 42(84 \%), 41(82 \%)$ and $34(68 \%)$ cases in the common femoral, superficial femoral, deep femoral and popliteal arterial segments respectively while the CT angiography was able to detect $42(84 \%), 45(90 \%), 46(92 \%)$ and $38(76 \%)$ cases in the common femoral, superficial femoral, deep femoral and popliteal arterial segments respectively. Detection rate for grade- 1 and grade- 2 stenosis collectively by CDUS and CTA is $25(12.5 \%)$ and $10(5 \%)$ respectively. The detection rate for Grade-3 by CDUS is $8(4 \%)$ and $11(5.5 \%)$ respectively and Grade 4 stenosis was similar for both CDUS and CTA in the mentioned arterial segments of the femoro-popliteal group.

A total of 200 arterial segments were examined in the tibio-peroneal region and following were the observations (table no-3): Using CDUS, we were not able to delineate 6 arterial segments in the leg due to the gas gangrene. No abnormality (Grade 0 stenosis) detected in 28(56\%), 30(62.5\%), $32(66.67 \%)$ and $32(62.5 \%)$ cases in the tibio-peroneal trunk, anterior tibial, posterior tibial and peroneal arterial segments respectively while the CT angiography demonstrated no abnormality in $28(56 \%), 30(60 \%), 32(64 \%)$ and $38(76 \%)$ cases in the tibio-peroneal, anterior tibial, posterior tibial and peroneal arterial segments respectively.

Detection rate for grade- 1 and grade- 2 stenosis by CDUS and CTA is 55(28.3\%) and 36(18\%) respectively. The detection rate for the Grade-3 and Grade 4 stenosis is $17(8.7 \%)$ and $29(14.5 \%)$ by CDUS and CTA respectively in the distal arterial segments. Few arterial segments (In two patients) were not visible due to the gangrenous changes in the leg with associated subcutaneous emphysema obscuring the field for the sound waves using CDUS. Collectively the Colour Doppler Ultrasonography detected no abnormality in $121(80.6 \%), 159(79.5 \%)$ and $122(61 \%)$ segments in the aorto-iliac, femoro-popliteal and tibial arterial segments respectively as normal (Grade 0 stenosis), while the CT angiography was able to detect $129(86 \%), 171(85.5 \%)$ and $135(67.5 \%)$ cases in the aorto-iliac, femoro-popliteal and tibial arterial segments respectively as normal. The detection rate for the Grade 1 and 2 stenosis by CDUS and CTA is $94(17.27 \%)$ and 52(9.45\%) respectively in all the three arterial segments.

(Table no- 4). Greater number of the arterial segments with Grade- 3 stenosis was detected by the CTA in the femoro-popliteal and tibio-peroneal region than the CDUS, however both CDUS and CTA detected equal segments with Grade- 3 stenosis in aorto-iliac group. Both CDUS and CTA were able to detect all the Grade -4 stenosis segments in the aorto-iliac and femoro-popliteal region. CTA was able to quantify more number of segments in the tibio-peroneal region than CDUS due to the non-visibility of the arterial segments because of gas gangrene. Further the arterial segments were divided into the following segments: 1) Supra-popliteal, 2) Infra-popliteal.

It was noted that the CDUS was able to detect more number of the stenotic arterial segments in the both supra-popliteal and infra-popliteal region than CTA. In supra-popliteal segment $70(20.9 \%)$ segments were picked by CDUS in comparison to the $50(14.2 \%)$ segments by CTA, while in infrapopliteal segment $72(36 \%)$ segments were picked by CDUS in comparison to the $65(32.5 \%)$ segments by CTA. Early stenotic lesions were better picked by the CDUS whereas Grade3 and 4 lesions were better demonstrated by the CTA. (Graph no-1) Collectively in all segments the CDUS is able to detect $142(25.8 \%)$ positive cases, in comparison to the CTA that is $115(20.9 \%)$, i.e 5.2\% more no. of positive cases. Also, it scores over CTA in detection of the Grade 1 and 2 stenoses. The number of segments with greater than $50 \%$ stenosis were $48(8.7 \%)$ and $63(11.45 \%)$ by CDUS and CTA respectively. 
The number of segments with less than 50\% stenosis were $94(17.1 \%)$ and $52(9.4 \%)$ by CDUS and CTA respectively (Table no-5).Calcified plaques noted in the arterial segments were noted on both CDUS and CTA. More number of the calcified plaques was noted on CTA than CDUS. (Graph no-2).

DISCUSSION: The present study was carried out to find out a better diagnostic test out of the CDUS and CTA. The imaging characteristics were recorded in all patients and documented. The results were analysed, studied and compared with similar studies. A total of 550 vessel segments were imaged by both DUS and MDCTA in 50 patients. Due to the gangrenous changes in the leg few arterial segments in the leg were not visualized in the CDUS but were clearly demarcated in the CTA. Rest all arterial segments were successfully analyzed by both modalities. We used a different grading system for the degree of stenosis i.e. Grade 0/ Normal - 0 \%, Grade I - 0-25\%, Grade II - 25-50\%, Grade III - 50-75\% and Grade IV -> 75\%(including occlusion) in comparison to that was proposed by de Smet AA et al. ${ }^{1}$ in 1996 and adopted by. Ofer et al. ${ }^{2}$ in 2003 also used different grading scale for the stenosis.

Through CDUS we were able to label 46(92\%), 36(72\%) and 39(78\%) cases in the infrarenal aorta, common iliac and external iliac arterial segments respectively as normal (Grade 0 stenosis). However, the CT angiography was able to quantify $49(98 \%), 39(78 \%)$ and $41(82 \%)$ cases in the infrarenal aorta, common iliac and external iliac arterial segments respectively as normal (Grade 0 stenosis). The detection rate for the Grade 4 stenosis was similar for both CDUS and CTA in the mentioned arterial segments of the aorto-iliac group. More number of the cases with Grade I stenosis were detected by CDUS than CTA in the aorto-iliac region. Colour Doppler Ultrasonography detected no abnormality in $42(84 \%), 42(84 \%), 41(82 \%)$ and $34(68 \%)$ cases in the common femoral, superficial femoral, deep femoral and popliteal arterial segments respectively as normal (Grade 0 stenosis).

However, the CT angiography was able to detect 42(84\%), 45(90\%), 46(92\%) and 38(76\%) cases in the common femoral, superficial femoral, deep femoral and popliteal arterial segments respectively as normal. The detection rate for the Grade 4 stenosis/ occlusion was similar for both CDUS and CTA in the mentioned arterial segments of the femoro-popliteal region. Using CDUS, we were not able to delineate 6 arterial segments in the leg due to the gas gangrene.

CDUS detected no abnormality in 28(56\%), 30(62.5\%), 32(66.67\%) and 32(62.5\%) cases in the tibio-peroneal trunk, anterior tibial, posterior tibial and peroneal arterial segments respectively (Grade 0 stenosis). However, the CT angiography demonstrated no abnormality (Grade 0 stenosis) in $28(56 \%), 30(60 \%), 32(64 \%)$ and $38(76 \%)$ cases in the tibio-peroneal, anterior tibial, posterior tibial and peroneal arterial segments respectively. The detection rate for the Grade-3 and Grade 4 stenosis is more with the CTA in the distal arterial segments which were not visible due to the gangrenous changes in the leg with associated subcutaneous emphysema obscuring the field for the sound waves using CDUS. Colour Doppler Ultrasonography detected no abnormality in 121, 159 and 120 segments in the aorto-iliac, femoro-popliteal and tibial arterial segments respectively as normal (Grade 0 stenosis). However, the CT angiography was able to 129, 171 and 133 cases in the aorto-iliac, femoropopliteal and tibial arterial segments respectively as normal.

The detection rate for the Grade 1 and 2 stenoses was higher for CDUS than CTA in all the three arterial segments. Greater number of the arterial segments with Grade 3 stenosis was detected by the CTA in the femoro-popliteal and tibio-peroneal region than the CDUS. Both CDUS and CTA were able to detect all the Grade 4 stenosis segments in the aorto-iliac and femoro-popliteal region. 
However CTA was able to quantify more number of segments in the tibio-peroneal region than CDUS due to the non-visibility of the arterial segments because of gas gangrene.

It was noted that the CDUS was able to detect more number of the stenotic arterial segments in the both supra-popliteal and infra-popliteal region than CTA. In supra-popliteal segment 70(20.9\%) segments were picked by CDUS in comparison to the 50(14.2\%) segments by CTA. However, in infrapopliteal segment $72(36 \%)$ segments were picked by CDUS in comparison to the $65(32.5 \%)$ segments by CTA. Early stenotic lesions were better picked by the CDUS whereas Grade 3 and 4 lesions were better demonstrated by the CTA. It was noted that the CDUS was able to detect more number of the stenotic arterial segments in the both supra-popliteal and infra-popliteal region than CTA. Early stenotic lesions were better picked by the CDUS whereas Grade-3 and 4 lesions were better demonstrated by the CTA.

In our study, out of the total 550 vessel segments, CDUS is able to detect $142(25.8 \%)$ positive cases, in comparison to the CTA which was able to detect $115(20.9 \%)$, i.e. $5.2 \%$ more no. of positive cases. We were not able to image 6 segments in the infrapopliteal region due to the overlying subcutaneous emphysema due to gas gangrene. Kayhan A et al. ${ }^{3}$ in 2012 imaged 774 vessel segments in 43 patients by both CDUS and CTA. They were able to image all the segments. The total number of diseased segments found with CDUS was 86(11.1\%) versus $130(16.8 \%)$ identified by MDCTA. In present study we found more number of the cases of PAOD positive for stenosis with CDUS than CTA, in comparison to the study conducted by Kayhan A et al. ${ }^{3}$ in 2012. That is because of the ability of CDUS to detect more number of the segments with $<50 \%$ stenosis in early PAOD.

In present study the number of segments with greater than $50 \%$ stenosis were $48(8.7 \%)$ and 63(11.45\%) by CDUS and CTA respectively, while A. Kayhan et al. ${ }^{3}$ in 2012 found the number of segments with greater than $50 \%$ stenosis were $27(3.49 \%)$ and $35(4.52 \%)$ on DUS and MDCTA respectively. In respective to $>50 \%$ stenosis the results of our study is comparable to that of $A$. Kayhan et al. ${ }^{3}$ in 2012. Collins R, Granny G, Burch J et al ${ }^{4}$ in 2007 and Met R, Bipat S, Legemate D A et al. 5 in 2009 are also found CTA to be more accurate modality in assessing the presence and extent of peripheral arterial disease. The number of segments with less than $50 \%$ stenosis were $94(17.1 \%)$ and 52(9.4\%) as detected by CDUS and CTA respectively. Hatsukami TS et al 6 in 1992, Linke RJ et al. ${ }^{7}$ in 1994, Bergamini TM et al. ${ }^{8}$ in 1995, Sensier Y et al. ${ }^{9}$ in 1996 also found that CDUS as an accurate screening tool for the evaluation of the peripheral arterial disease. More number of calcified plaque are detected by CTA then CDUS. Joshi A, Nimbkar A, Merchant S, et al ${ }^{10}$ in 2004 also found CTA to be more accurate in detection of calcified plaque.

CONCLUSION: In all the cases of peripheral arterial disease, purpose of any diagnostic modality is to define the anatomy, evaluate the severity of disease, assess the distal circulation and identify unsuspected lesions. Here we have attempted to compare two non-invasive modalities of today's era which are now becoming easily available in urban as well as semiurban areas of India. The ultrasound findings providing information on the, Extent and severity of the disease, Soft tissue plaques, Thrombosis, Segmental flow analysis, Segmental length analysis, Collateral circulation, Recanalization Pre- and post-operative flow evaluation. There are few limitations as being operator dependency, time consumption, bowel gas, edema and obesity.

CT angiography on the other hand is less time consuming, minimally invasive requiring only an intravenous injection of contrast medium but also involves radiation exposure and an inherent risk of allergic reactions. Multiplanar reconstruction aids in the visualization of segment length of 
stenosis, better appreciation of collateral circulation and better reproduction of the arterial tree. Soft tissue plaques, early recanalization, post-op flow evaluation and co-morbid conditions (renal disease) being important limitations of CT angiography. Doppler sonography is better than CT angiography in the diagnosis of early onset(Grade I \& II) cases, with a better assessment of soft plaques, segmental flow and re-canalisation in peripheral arterial disease while CTA is more accurate in sever stenosis(Grade-3\&4), near occlusion and complete occlusion stage.

\section{BIBLIOGRAPHY:}

1. Bergamini TM, Tatum CM Jr, Marshall C, Hall-Disselkamp B, Richardson JD. Effect of multilevel sequential stenosis on lower extremity arterial duplex scanning. Am J Surg. 1995 Jun; 169(6):564-6.

2. Collins R, Cranny G, Burch J, Aguiar-Ibáñez R, Craig D, Wright K, Berry E, Gough M, Kleijnen J, Westwood M A systematic review of duplex ultrasound, magnetic resonance angiography and computed tomography angiography for the diagnosis and assessment of symptomatic, lower limb peripheral arterial disease Health Technology Assessment 2007; Vol. 11: No. 20.

3. De Smet AA, Ermers EJ, Kitslaar PJ Duplex velocity characteristics of aortoiliac stenoses. J Vasc Surg. 1996 Apr; 23(4):628-36.

4. Hatsukami TS, Primozich JF, Zierler RE, Harley JD, Strandness DE. Jr. ColorDoppler imaging of infrainguinal arterial occlusive disease. J Vasc Surg 1992; 16:527-33.

5. Joshi A, Nimbkar V, Merchant S, Mhashelkar Y, Talekar K Role of CT angiography in the evaluation of peripheral vasculature using MSCT - our initial experience. Indian J Radiol Imaging 2004; 14:309-15.

6. Kayhana A, Palabiyikb F, Serinsozb S, Kiris A, Bayramoglu S, Williamsc J T B, Cimilli T. Multidetector CT angiography versus arterial duplex USG in diagnosis of mild lower extremity peripheral arterial disease: Is multidetector CT a valuable screening tool? European Journal of Radiology 81(2012) 542- 546.

7. Linke RJ, Davies RP, Giles AJ, Walsh JA, Thompson BW. Colour duplex ultrasound: a screening modality for femoropopliteal disease in patients with intermittent claudication. Australas Radiol 1994; 38:320-3.

8. Met R, Bipat S, Legemate DA, Reekers JA, Koelemay MJ.Diagnostic performance of computed tomography angiography in peripheral arterial disease: a systematic review and meta-analysis. JAMA. 2009 Jan 28; 301(4):415-24.

9. Ofer A, Nitecki SS, Linn S, Epelman M, Fischer D, Karram T, Litmanovich D, Schwartz H, Hoffman A, Engel A. Multidetector CT Angiography of Peripheral Vascular Disease: A Prospective Comparison with Intraarterial Digital Subtraction Angiography AJR 2003;180:719-724.

10. Sensier Y, Hartshorne T, Thrush A, Handford H, Nydahl S, London NJ. The effect of adjacent segment disease on the accuracy of colour duplex scanning for the diagnosis of lower limb arterial disease. Eur J Vasc Endovasc Surg 1996; 12:238-42. 


\section{ORIGINAL ARTICLE}

Table No. 1: Stenosis severity grade in CDUS and CTA in infrarenal aorta Common iliac artery, External iliac artery.

\begin{tabular}{|c|c|c|c|c|c|}
\hline \multicolumn{6}{|c|}{ Stenosis Severity Grade } \\
\hline Modality & 0 & 1 & 2 & 3 & 4 \\
\hline & \multicolumn{7}{|c|}{ Infrarenal Aorta } \\
\hline CDUS & 46 & 3 & 1 & 0 & 0 \\
\hline CTA & 49 & 0 & 1 & 0 & 0 \\
\hline & \multicolumn{7}{|c|}{ Common Iliac Artery } \\
\hline CDUS & 36 & 4 & 2 & 1 & 7 \\
\hline CTA & 39 & 2 & 1 & 1 & 7 \\
\hline \multicolumn{7}{|c|}{ External Iliac Artery } \\
\hline CDUS & 39 & 2 & 2 & 0 & 7 \\
\hline CTA & 41 & 0 & 2 & 0 & 7 \\
\hline \multicolumn{7}{|c|}{ Table: 1} \\
\hline
\end{tabular}

Table No 2: Stenosis severity grade in CDUS and CTA in Common femoral, superficial femoral artery, Deep femoral artery, and Popliteal artery.

\begin{tabular}{|c|c|c|c|c|c|}
\hline \multicolumn{6}{|c|}{ Stenosis Severity Grade } \\
\hline Modality & 0 & 1 & 2 & 3 & 4 \\
\hline & Common Femoral Artery \\
\hline CDUS & 42 & 3 & 0 & 3 & 2 \\
\hline CTA & 42 & 3 & 0 & 3 & 2 \\
\hline & Superficial Femoral Artery \\
\hline CDUS & 42 & 3 & 2 & 1 & 2 \\
\hline CTA & 45 & 0 & 1 & 2 & 2 \\
\hline & \multicolumn{7}{|c|}{ Deep Femoral Artery } \\
\hline CDUS & 41 & 5 & 2 & 1 & 1 \\
\hline CTA & 46 & 0 & 1 & 2 & 1 \\
\hline & 34 & 4 & 6 & 3 & 3 \\
\hline CDUS & 34 & 0 & 5 & 4 & 3 \\
\hline CTA & 38 & 0 & \\
\hline & \multicolumn{7}{|c|}{ Table: 2} \\
\hline
\end{tabular}




\section{ORIGINAL ARTICLE}

Table No. 3: Stenosis severity grade in CDUS and CTA in Tibio- peroneal trunk, ATA, PTA, Peroneal artery.

\begin{tabular}{|c|c|c|c|c|c|}
\hline \multicolumn{7}{|c|}{ Stenosis Severity Grade } \\
\hline Modality & 0 & 1 & 2 & 3 & 4 \\
\hline & \multicolumn{7}{|c|}{ Tibio-peroneal trunk } \\
\hline CDUS(100\%) & 28 & 7 & 9 & 4 & 2 \\
\hline CTA(100\%) & 30 & 5 & 6 & 7 & 2 \\
\hline & \multicolumn{7}{|c|}{ Anterior Tibial Artery } \\
\hline CDUS(96\%) & 30 & 6 & 7 & 3 & 2 \\
\hline CTA(100\%) & 33 & 3 & 6 & 5 & 3 \\
\hline & \multicolumn{7}{|c|}{ Posterior Tibial Artery } \\
\hline CDUS(96\%) & 32 & 5 & 7 & 3 & 1 \\
\hline CTA(100\%) & 34 & 3 & 6 & 4 & 3 \\
\hline \multicolumn{7}{|c|}{ Peroneal artery } \\
\hline CDUS(96\%) & 32 & 8 & 6 & 2 & 0 \\
\hline CTA(100\%) & 38 & 2 & 5 & 3 & 2 \\
\hline
\end{tabular}

Table No. 4: Stenosis severity grade in CDUS and CTA in Aorta-iliac region, Femoropoplitial region and Tibial region.

\begin{tabular}{|c|c|c|c|c|c|c|}
\hline \multicolumn{6}{|c|}{ Stenosis Severity Grade } & \\
\hline Modality & 0 & 1 & 2 & 3 & 4 & \\
\hline & \multicolumn{5}{|c|}{ Aorto-iliac region } & \\
\hline CDUS & 121 & 9 & 5 & 1 & 14 & 29 \\
\hline \multirow[t]{2}{*}{ CTA } & 129 & 2 & 4 & 1 & 14 & 21 \\
\hline & \multicolumn{5}{|c|}{ Femoro-popliteal region } & \\
\hline CDUS & 159 & 15 & 10 & 8 & 8 & 41 \\
\hline \multirow[t]{2}{*}{ CTA } & 171 & 3 & 7 & 11 & 8 & 29 \\
\hline & \multicolumn{5}{|c|}{ Tibial region } & \\
\hline CDUS & 122 & 26 & 29 & 12 & 5 & 72 \\
\hline CTA & 135 & 13 & 23 & 19 & 10 & 65 \\
\hline
\end{tabular}




\section{ORIGINAL ARTICLE}

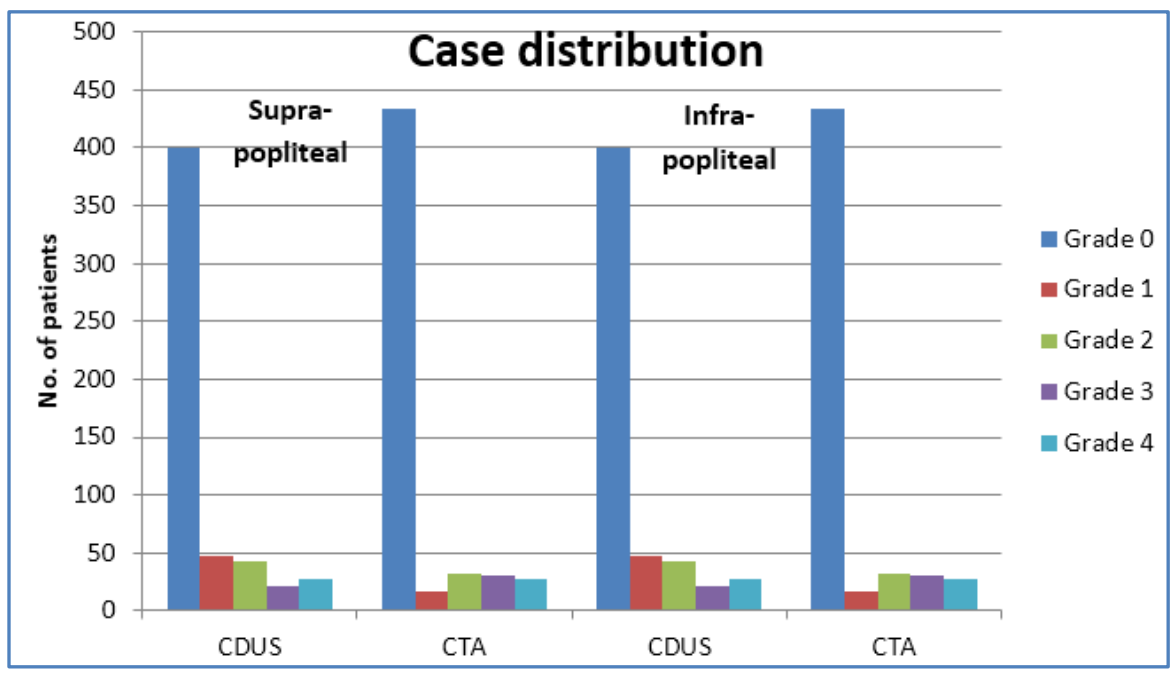

Graph 1: Stenosis severity grade in CDUS and CTA in suprapopliteal and infrapopliteal arteries

Table No. 5: Stenosis severity grade in CDUS and CTA in all arterial segments.

\begin{tabular}{|c|c|c|c|c|c|c|}
\hline \multicolumn{6}{|c|}{ Stenosis Severity Grade } & \multirow[b]{2}{*}{ Total positive } \\
\hline Modality & 0 & 1 & 2 & 3 & 4 & \\
\hline & \multicolumn{5}{|c|}{ Peripheral Arteries } & \\
\hline CDUS & 402 & 50 & 44 & 21 & 27 & $142(25.8 \%)$ \\
\hline CTA & 435 & 18 & 34 & 31 & 32 & 115(20.9\%) \\
\hline
\end{tabular}

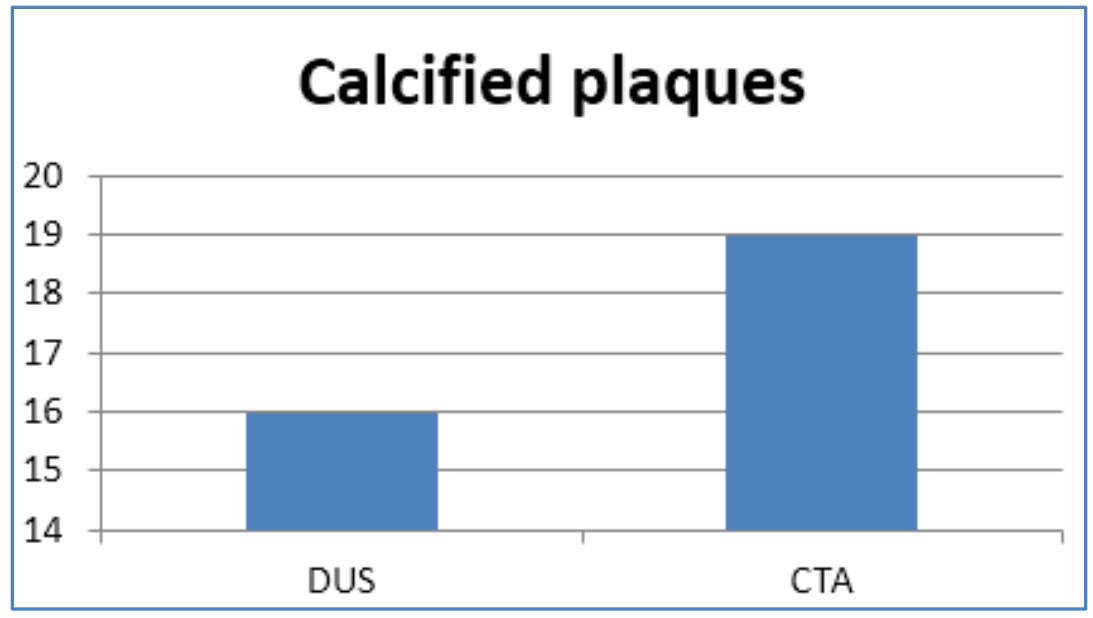

Graph No 2: Calcified plaques detected in CDUS and CTA 


\section{ORIGINAL ARTICLE}

\section{AUTHORS:}

1. Satyabhuwan Singh Netam

2. Rajesh Singh

3. Sanjay Kumar

4. Atees Singhal

5. Vishal Jain

\section{PARTICULARS OF CONTRIBUTORS:}

1. Associate Professor Department of Radiodiagnosis Pt. J. N. M. Medical College, Raipur, Chhattisgarh.

2. Assistant Professor Department of Radiodiagnosis Pt. J. N. M. Medical College, Raipur, Chhattisgarh.

3. Associate Professor Department of Radiodiagnosis Pt. J. N. M. Medical College Raipur, Chhattisgarh.

FINANCIAL OR OTHER COMPETING INTERESTS: None
4. Junior Resident (PG), Department of Radiodiagnosis Pt. JNM Medical College, Raipur, C. G, India

5. Assistant Professor, Department of Radiodiagnosis Pt. JNM Medical College, Raipur, C. G, India.

\section{NAME ADDRESS EMAIL ID OF THE} CORRESPONDING AUTHOR:

Satyabhuwan Singh Netam,

D-20, Avani, Vihar, Daldalseoni Road,

Mova, Raipur, Chhattisgarh.

E-mail: sbsnetam@yahoo.com

Date of Submission: 25/09/2015.

Date of Peer Review: 26/09/2015.

Date of Acceptance: 06/10/2015.

Date of Publishing: 14/10/2015. 